\title{
Analytical Solution for Pressure-Coupled Combustion Response Functions of Composite Solid Propellants
}

\author{
Michael Shusser* and Fred E. C. Culick \\ California Institute of Technology, Pasadena, California 91125 \\ and \\ Norman S. Cohen \\ Cohen Professional Services, Los Angeles, California 90035
}

DOI: $\underline{10.2514 / 1.21502}$

\begin{abstract}
This paper extends the classical analytical solution for small perturbation analysis of the pressure-coupled response of a homogeneous propellant to any two-component composite propellant. The solution obtained is general and can be used with any particular model for propellant combustion. As an example, the Cohen and Strand ammonium perchlorate propellant model for a single ammonium perchlorate particle size was used in this work. The results and their mechanistic significance are presented and discussed. It is shown that, for a two-component composite propellant, two forms of pressure exponents arise from the analysis. The significance of the second exponent is that it enables the composite propellant to be viewed as a homogeneous propellant with a frequencydependent exponent via the coupling coefficients. It is found that the ammonium perchlorate is the main source of instability because of its condensed phase exothermicity and monopropellant flame kinetics. This will be a problem with energetic materials in general. The inert binder provides a stabilizing influence because of its endothermicity and the diffusion flame formed with the ammonium perchlorate. Effects of ammonium perchlorate particle size and pressure stem from the changing flame structure and its effect on burning rate.
\end{abstract}

\section{Nomenclature}

$A=$ dimensionless parameter characteristic of surface decomposition

$B=$ dimensionless parameter characteristic of heat feedback law

$C=$ coupling parameter

$D=$ defined by Eqs. (23) and (24)

$F=$ classical response function, Eq. (17)

$f \quad=$ frequency of oscillations

$n=$ burn rate pressure exponent

$n^{\prime} \quad=$ defined by Eq. $(\underline{49})$

$p \quad=$ pressure

$R \quad=\quad$ response function

$r \quad=$ burning rate

$S=$ fraction of propellant surface area occupied by a component

$T=$ temperature

$T_{0} \quad=\quad$ bulk temperature

$t=$ time

$x=$ distance (negative into the solid)

$z=$ dimensionless distance

$\alpha=$ fraction of propellant mass flow rate from ammonium perchlorate

$\delta=\Omega_{2} / \Omega_{1}$

$\theta=$ dimensionless temperature

$\kappa=$ thermal diffusivity

$\lambda=$ ammonium perchlorate complex frequency

Presented as Paper 3426 at the 37th AIAA/ASME/SAE/ASEE Joint Propulsion Conference and Exhibit, Salt Lake City, UT, 8-11 July 2001; received 1 December 2005; revision received 18 May 2008; accepted for publication 26 May 2008. Copyright (C) 2008 by the American Institute of Aeronautics and Astronautics, Inc. All rights reserved. Copies of this paper may be made for personal or internal use, on condition that the copier pay the $\$ 10.00$ per-copy fee to the Copyright Clearance Center, Inc., 222 Rosewood Drive, Danvers, MA 01923; include the code 0748-4658/08 $\$ 10.00$ in correspondence with the CCC.

*Postdoctoral Scholar; currently Faculty of Mechanical Engineering, Technion, 32000 Haifa, Israel. Member AIAA.

${ }^{\dagger}$ Professor of Mechanical Engineering and Jet Propulsion. Fellow AIAA.

${ }^{\ddagger}$ Subcontractor. Associate Fellow AIAA.

$\begin{array}{lll}\mu & = & \text { binder complex frequency } \\ \rho & = & \text { density } \\ \tau & = & \text { dimensionless time } \\ \Omega & = & \text { dimensionless frequency }\end{array}$

Subscripts

$\begin{array}{lll}f & = & \text { binder } \\ \operatorname{Im} & = & \text { imaginary part } \\ \text { prop } & = & \text { propellant } \\ \operatorname{Re} & = & \text { real part } \\ s & = & \text { surface value } \\ x & = & \text { oxidizer } \\ 1 & = & \text { oxidizer } \\ 2 & = & \text { binder }\end{array}$

Superscripts

$\begin{array}{lll}(\mathrm{Im}) & = & \text { imaginary part } \\ (\mathrm{Re}) & = & \text { real part } \\ & = & \text { perturbations }\end{array}$

\section{Introduction}

$\mathbf{U}$ NDERSTANDING of unsteady combustion of solid propellants is a basis for analyzing, predicting, and suppressing of combustion instabilities in solid rocket motors. The main cause of these instabilities is the response of the combustion process to pressure oscillations in the combustion chamber. Hence, the most important physical quantity characterizing unsteady combustion is the response of mass burning rate to pressure oscillations.

The simplest models of the unsteady combustion of solid propellants [quasi-steady homogeneous one-dimensional (QSHOD) models [1]] assume that the propellant is homogeneous, the gas phase is quasi steady, and the solid phase is one-dimensional. This approximation results in the well-known classical analytical solution for small perturbation analysis of a homogeneous propellant [2]. If one defines the pressure-coupled response function $R_{p}$

$$
R_{p}=\frac{r^{\prime}}{\bar{r}} \frac{\bar{p}}{p^{\prime}}
$$


where $\bar{r}, \bar{p}$ are steady-state values (overbars denote steady-state values throughout the paper) of burning rate and pressure, and $r^{\prime}, p^{\prime}$ are their perturbations, respectively, then

$$
R_{p}=\frac{n A B}{\lambda+A / \lambda-1-A(1-B)}
$$

Here, $\lambda$ is a nondimensional complex frequency of oscillations, whereas each of the basic parameters $n, A$, and $B$ characterize the dependence of the steady-state burning rate $\bar{r}$ on a certain physical quantity. Thus, the pressure exponent $n$ characterizes the dependence of $\bar{r}$ on pressure, $A$ (characteristic of the surface decomposition) is related to the dependence on the surface temperature, and $B$ (characteristic of the heat feedback law) shows the dependence on the surface temperature gradient, that is, heat flux.

The classical solution has been used extensively in combustion instability studies. It is, however, limited to homogeneous propellants, whereas most modern solid propellants are composite propellants. To model composite propellant combustion, one must account for the heterogeneity and two-dimensionality of the problem. Following the classical work of Nachbar [3] , a widely used approach has been to describe these effects approximately by using a "sandwich" model. This model represents a composite structure as side-by-side slabs of fuel and oxidizer. Steady burning of such sandwiches has been the object of numerous studies [4].

Cohen and Strand perturbed their steady-state model [5] to describe the nonsteady combustion of a composite propellant [ $[\underline{6}, 7]$. They were able to calculate the linearized combustion response and to explain effects of ammonium perchlorate (AP) particle size, pressure, and crossflow on combustion response properties. Shusser et al. [8] used a sandwich model to numerically compute nonlinear combustion response properties of a composite propellant by solving the heat conduction equation in each component, with the Cohen and Strand model [5] as a boundary condition. A similar approach was used by Rasmussen and Frederick [9] and Surzhikov et al. [10].

Recently, a combustion model based on calculating the burning of a three-dimensional array of randomly packed spheres was proposed $[11,12]$. This model is first to enable detailed consideration of the microstructure of the propellant. However, as with simpler sandwich models, the predictive value of the model is limited by the approximate constitutive relations it uses. Moreover, the need for computationally fast models remains for future coupling of the propellant combustion with the gas dynamics of the combustion chamber in comprehensive motor analyses.

It is well recognized that closed-form solutions are very useful, as they provide insights not otherwise available. A general analytic solution for the response function of composite propellants has not yet been obtained. Previous attempts to use the classical solution (2) for AP composite propellants were unsuccessful. For example, Brown and Muzzy [13], who tried to use solution (2) to match their experiments, obtained physically unrealistic values of the parameters.

The reason for this failure is obvious. Although a total propellant burning rate exists, surface temperature and heat flux are different for the AP and the binder components of the propellant. There are no "average" or "total" surface temperatures or surface temperature gradients and, therefore, no $A$ and $B$ parameters for the propellant as a whole can be defined.

In the present work, we obtain the first closed-form analytical solution for a heterogeneous unsteady model of composite propellants. $\stackrel{\S}{\S}$ Such solution can serve several purposes. First, it can give more insight into the physics of the process. It also provides a better way to see trends with changes in physical conditions, such as pressure or temperature. It can be very helpful for adjusting parameters to achieve better modeling. Finally, it serves as a benchmark for numerical computations.

${ }^{\S}$ Though only pressure-coupled response is considered in this analytical paper, the model can be extended to include velocity coupling by including an appropriate dynamic erosive burning model, assuming that dynamic erosive burning is the essential mechanism. This was done in the numerical work [8].
In the next section, we will demonstrate how to obtain such an analytical solution for a composite propellant for a single (monomodal) particle size. For such a coupled two-component system, each component has its own set of basic parameters analogous to $A, B, n$, whereas two additional coefficients are due to the coupling between the oxidizer and the binder.

This solution is general in the sense that it is not limited to any particular ingredient or model for propellant combustion. A choice of a specific model influences only the values of the parameters, as they are obtained by linearizing the model. In Sec. III, we calculate the coefficients for the Cohen and Strand AP propellant model [5-7], show how they change with pressure and particle size, and compare the analytical solution with numerical computations (the numerical scheme has been described elsewhere [8]). We then proceed with a parametric study of the general solution in Sec. IV. Intrinsic instability is considered in Sec. $\mathrm{V}$. The pressure exponent of a composite propellant is studied in Sec. VI.

\section{Analytical Solution}

The physical model used for the analysis is the classical AP/binder sideways sandwich. The approach yields a system of two unsteady one-dimensional heat conduction equations coupled through their boundary conditions at the solid surface. Their linearized form, which is appropriate for small perturbation analysis, is as follows:

$$
\begin{aligned}
& \frac{\partial \theta_{1}^{\prime}}{\partial \tau_{1}}+\frac{\partial \theta_{1}^{\prime}}{\partial z_{1}}=\frac{\partial^{2} \theta_{1}^{\prime}}{\partial z_{1}^{2}}-\frac{r_{1}^{\prime}}{\bar{r}_{1}} e^{z_{1}} \\
& \frac{\partial \theta_{2}^{\prime}}{\partial \tau_{2}}+\frac{\partial \theta_{2}^{\prime}}{\partial z_{2}}=\frac{\partial^{2} \theta_{2}^{\prime}}{\partial z_{2}^{2}}-\frac{r_{2}^{\prime}}{\bar{r}_{2}} e^{z_{2}}
\end{aligned}
$$

Here, the subscripts 1,2 relate to the API and the binder, respectively, the overbar denotes steady-state values, and the primed quantities are their perturbations. The dimensionless temporal and spatial coordinates $\tau_{1}, \tau_{2}, z_{1}, z_{2}$, and temperature perturbations $\theta_{1}^{\prime}, \theta_{2}^{\prime}$ are defined by

$$
\begin{array}{cc}
z_{1}=\frac{\bar{r}_{1} x}{\kappa_{1}} ; & z_{2}=\frac{\bar{r}_{2} x}{\kappa_{2}} \\
\tau_{1}=\frac{\bar{r}_{1}^{2} t}{\kappa_{1}} ; & \tau_{2}=\frac{\bar{r}_{2}^{2} t}{\kappa_{2}} \\
\theta_{1}^{\prime}=\frac{T_{1}-\bar{T}_{1}}{\bar{T}_{1 s}-T_{0}} ; & \theta_{2}^{\prime}=\frac{T_{2}-\bar{T}_{2}}{\bar{T}_{2 s}-T_{0}}
\end{array}
$$

Here, $x$ is the distance from the solid surface (negative into the solid), $t$ is the time, $\kappa_{1}$ and $\kappa_{2}$ are AP and binder thermal diffusivities, $T_{1 s}$ and $T_{2 s}$ are AP and binder surface temperatures, and $T_{0}$ is the temperature in the bulk of the solid.

The classical theory [2] uses the following boundary condition at the solid surface of a homogeneous propellant:

$$
z=0 \quad \frac{\partial \theta^{\prime}}{\partial z}=n B \frac{p^{\prime}}{\bar{p}}+\left(1-B+\frac{1}{A}\right) \frac{r^{\prime}}{\bar{r}}
$$

Following the classical formulation [2], we write the boundary conditions at the solid surface and the relationships between burning rate and temperature perturbations as

$$
z_{1}=0 \quad \frac{\partial \theta_{1}^{\prime}}{\partial z_{1}}=n_{x} B_{x} \frac{p^{\prime}}{\bar{p}}+\left(1-B_{x}+\frac{1}{A_{x}}\right) \frac{r_{1}^{\prime}}{\bar{r}_{1}}+C_{x} \frac{r_{2}^{\prime}}{\bar{r}_{2}}
$$

\footnotetext{
"We use AP to represent the oxidizer, but the generality of the approach is applicable to any two-component system.
} 


$$
\begin{gathered}
z_{2}=0 \quad \frac{\partial \theta_{2}^{\prime}}{\partial z_{2}}=n_{f} B_{f} \frac{p^{\prime}}{\bar{p}}+C_{f} \frac{r_{1}^{\prime}}{\bar{r}_{1}}+\left(1-B_{f}+\frac{1}{A_{f}}\right) \frac{r_{2}^{\prime}}{\bar{r}_{2}} \\
\frac{r_{1}^{\prime}}{\bar{r}_{1}}=\left.A_{x} \theta_{1}^{\prime}\right|_{z_{1}=0} ; \quad \frac{r_{2}^{\prime}}{\bar{r}_{2}}=\left.A_{f} \theta_{2}^{\prime}\right|_{z_{2}=0}
\end{gathered}
$$

One sees that now both AP and binder have their own set of basic parameters $n, A, B$, describing the relations between corresponding surface temperatures, heat fluxes, and burning rates. The $n_{x}$ and $n_{f}$ arise from the burning of the composite propellant: $n_{x}$ is not the value for AP by itself as a monopropellant, and for the inert binder by itself, $n_{f}$ would be meaningless. Their meaning will be discussed in Sec. VI. The coupling coefficients $C_{x}, C_{f}$ characterize how each heat flux is influenced by the other component's burning rate. Physically, they are caused mainly by the coupling through the multiple flame structure.

It should be noted that, due to the coupling, each component (AP or binder) behaves differently when in a propellant and when by itself. Thus, the values of the AP parameters $A_{x}, B_{x}$ will also be different from those for pure AP [14], as we shall see in the next section. We emphasize that the values of the parameters cannot be obtained by considering each component separately. They are determined by the physics of unsteady combustion of a composite propellant and can be obtained from its suitable model.

To complete the statement of the problem, we add the boundary conditions in the bulk of the solid

$$
z_{1} \rightarrow-\infty \quad \theta_{1}^{\prime}=0 ; \quad z_{2} \rightarrow-\infty \quad \theta_{2}^{\prime}=0
$$

We now impose on the system an oscillatory pressure perturbation of frequency $f$. Defining two dimensionless oscillatory frequencies

$$
\Omega_{1}=\frac{2 \pi f \kappa_{1}}{\bar{r}_{1}^{2}} ; \quad \Omega_{2}=\frac{2 \pi f \kappa_{2}}{\bar{r}_{2}^{2}}
$$

and AP and binder response functions

$$
R_{x}=\frac{r_{1}^{\prime}}{\bar{r}_{1}} \frac{\bar{p}}{p^{\prime}} ; \quad R_{f}=\frac{r_{2}^{\prime}}{\bar{r}_{2}} \frac{\bar{p}}{p^{\prime}}
$$

one obtains (the details can be found elsewhere [15])

$$
\begin{aligned}
& R_{x}=\frac{n_{x} B_{x}+C_{x} F_{f}}{\left[\lambda / A_{x}+1 / \lambda-\left(1-B_{x}\right)-1 / A_{x}\right]-\left(C_{x} C_{f} / n_{f} B_{f}\right) F_{f}} \\
& R_{f}=\frac{n_{f} B_{f}+C_{f} F_{x}}{\left[\mu / A_{f}+1 / \mu-\left(1-B_{f}\right)-1 / A_{f}\right]-\left(C_{x} C_{f} / n_{x} B_{x}\right) F_{x}}
\end{aligned}
$$

Here, $F$ is shorthand for the classical response function (2)

$$
F_{x}=\frac{n_{x} A_{x} B_{x}}{\lambda+A_{x} / \lambda-1-A_{x}\left(1-B_{x}\right)}
$$

with a corresponding formula for $F_{f}$. The complex frequencies $\lambda, \mu$ are obtained from

$$
\lambda^{2}-\lambda-i \Omega_{1}=0 ; \quad \mu^{2}-\mu-i \Omega_{2}=0
$$

One sees from Eqs. (15) and (16) that, when one of the coupling coefficients vanishes, the corresponding response function reduces to its homogeneous form [Eq. (2)]:

$$
R_{x}=F_{x} \quad \text { for } C_{x}=0 ; \quad R_{f}=F_{f} \quad \text { for } C_{f}=0
$$

The real and imaginary parts of $R_{x}$ and $R_{f}$ are written out in the Appendix.

\section{Cohen and Strand Model for Constitutive Combustion Relations}

The solution obtained in the previous section depends on several parameters. The values of the parameters can be determined by a particular model for propellant combustion. We have used the Cohen and Strand model [5] for this purpose, as was verified by incorporating it as the constituent combustion model in our response function work and comparing numerical solutions of the present model with a large family of response function data in [8]. Thus, to estimate the physically reasonable values of the combustion response parameters, we are justified in using the Cohen and Strand model []] to first calculate the coefficients.

The way to derive the eight coefficients $A_{x}, A_{f}, B_{x}, B_{f}, n_{x}, n_{f}, C_{x}$, $C_{f}$ is to linearize the nonlinear surface boundary condition as obtained from the original model (see [5-8]) and to compare its linearized form with Eqs. (9) and (10). The resulting detailed expressions are given elsewhere [15].

Figure 1 compares the analytical solution with numerical computations for the individual component AP and binder response functions as they behave in the composite propellant for a particular case. Both real and imaginary parts are shown in the figure. One sees that the agreement is very good.

We studied the influence of absolute pressure and particle size on the coefficients. AP concentration was kept at $87 \%$ throughout the calculations. Propellant bulk temperature was $25^{\circ} \mathrm{C}$. We show here only the main results. The details can be found in [15].

The $B_{x}$ parameter is shown in Fig. 2. We see immediately that $B_{x}$ in a propellant is much higher than in the classical formulation. Although the $B$ parameter usually lies between 0.6 and $1, B_{x}$ can be almost 3 . On the other hand, for high pressures and large particle sizes, $B_{x}$ becomes less than one; the reason for this is that, under these conditions, $C_{x} \approx 0$, as we shall see later, and hence the less stable AP is practically decoupled from the binder. $B_{x}$ decreases with increasing pressure mainly because of decreasing flame heights. The jump at about $80 \mathrm{~atm}$ for the $20 \mu \mathrm{m}$ case is caused by a change in the competing flame structure, as reflected in the $\beta_{F}$ model parameter (see $[5,16]$ for more details). ** $^{*}$

The binder parameters $A_{f}, B_{f}, n_{f}$ may not be compared with the classical homogeneous $A, B, n$ because an inert binder does not burn by itself. Here, we show only $B_{f}$ in Fig. $\underline{3}$. One sees that $B_{f}$ is even larger than $B_{x}$ and always stays above 2.4 . Thus, an inert binder is highly stabilizing to the AP. The calculations also demonstrate [15] that $n_{f}$ decreases much faster than $n_{x}$ with increasing pressure because the binder is heated only by the diffusion flame. AP is also heated by its monopropellant flame, which is the destabilizing influence, as can be inferred from our results in [14].

Figures $\underline{4}$ and $\underline{5}$ are plots of the coupling coefficients $C_{x}$ and $C_{f} . C_{x}$ is always negative and increases with pressure and particle size. This reflects the stabilizing influence of the binder on the AP by the coupling. The binder decomposition is endothermic and furnishes a diffusion flame. $C_{x}$ vanishes for high pressures and large sizes, where the AP becomes decoupled from the binder and behaves more like pure AP. The dependence of $C_{f}$ on pressure and particle size is more complicated, as seen from Fig. 5 . It is positive except for $2 \mu \mathrm{m}$ AP at low pressures. This means that the AP generally has a destabilizing influence on the binder when the binder is in fact burning. The AP monopropellant flame and exothermic condensed phase tend to be destabilizing. The analytical solution has served to clarify this for a composite propellant.

\section{Parametric Study}

A parametric study of the analytical solution [Eqs. (15) and (16)] is not easy because of the large number of parameters. The solution depends on eight coefficients $A_{x}, A_{f}, B_{x}, B_{f}, n_{x}, n_{f}, C_{x}, C_{f}$, and also

\footnotetext{
** It is possible that $B_{x}$ will decrease with decreasing pressure at very low pressures, $\ll 20 \mathrm{~atm}$, due to the importance of condensed phase exothermicity of the AP at low pressures $[\underline{8}, 14]$. However, the presence of the binder has a stabilizing influence on the $\overline{\mathrm{AP}}$.
} 


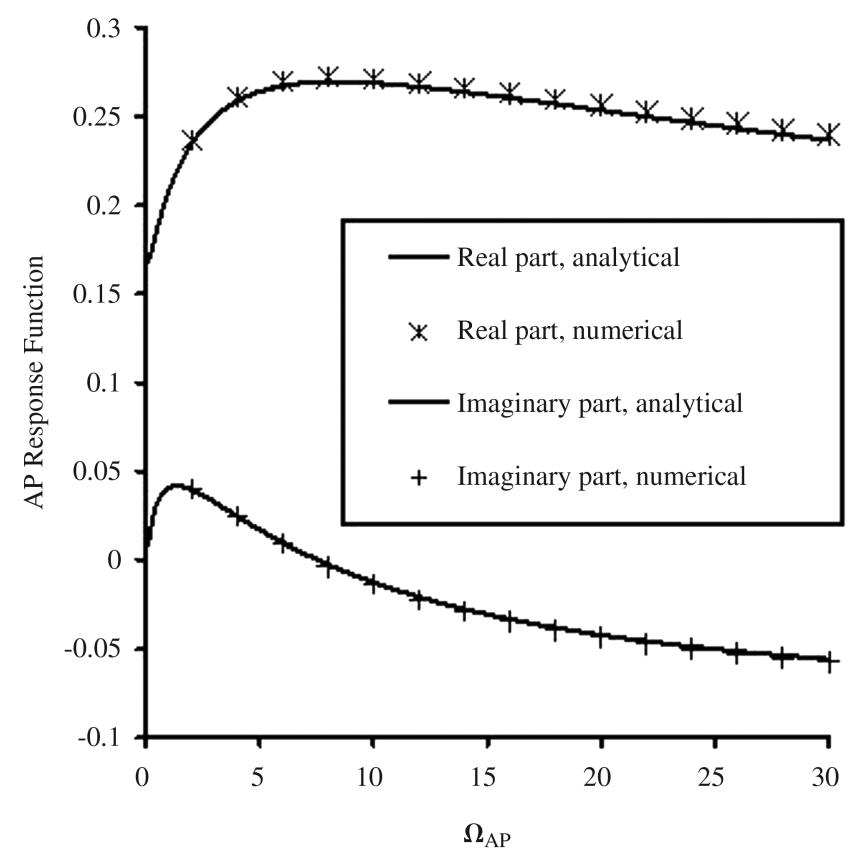

a)

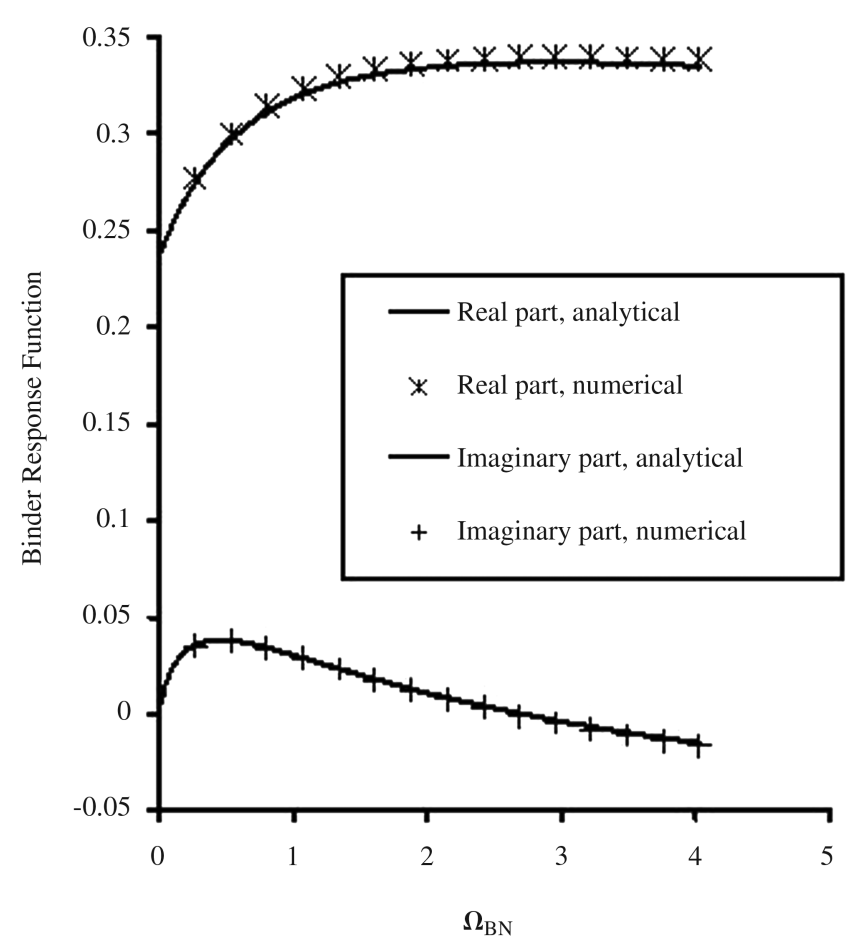

b)

Fig. 1 Comparison of analytical and numerical solution for composite propellant response function; $87 \% \mathrm{AP}(20 \mu \mathrm{m}) / \mathrm{hydroxyl}$-terminated polybutadiene, $68 \mathrm{~atm}, 298 \mathrm{~K}$ : a) AP response function, b) binder response function.

on the ratio of dimensionless frequencies that we will denote as $\delta$ :

$$
\delta=\Omega_{2} / \Omega_{1}
$$

We therefore adopt the following approach. We will choose reasonable basic values of each parameter from Sec. III results and vary only one of them at a time. Only the real part of the AP response function as it behaves in the propellant will be considered due to its being the most important driver for practical applications. Results of this exercise can provide guidance for more study in future work. The basic parameters chosen are given as follows: $A_{x}=12, B_{x}=1.5$, $n_{x}=1, A_{f}=8, B_{f}=3, n_{f}=0.8, C_{x}=-0.3, C_{f}=0.5, \delta=0.2$.

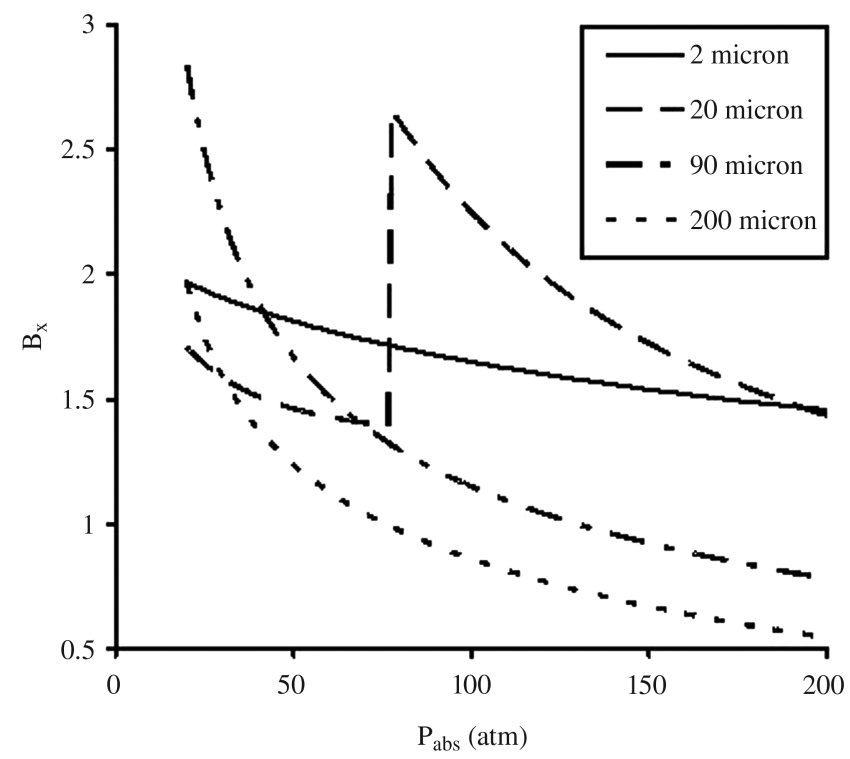

Fig. $2 B_{x}$ parameter for the Cohen and Strand model [ [ $]$.

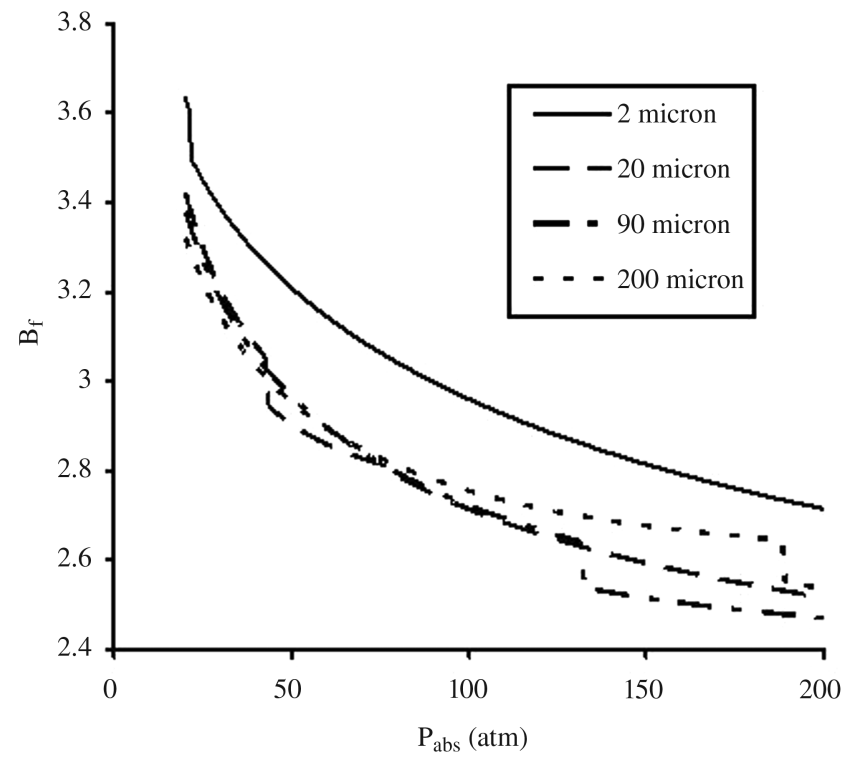

Fig. $3 B_{f}$ parameter for the Cohen and Strand model [5].

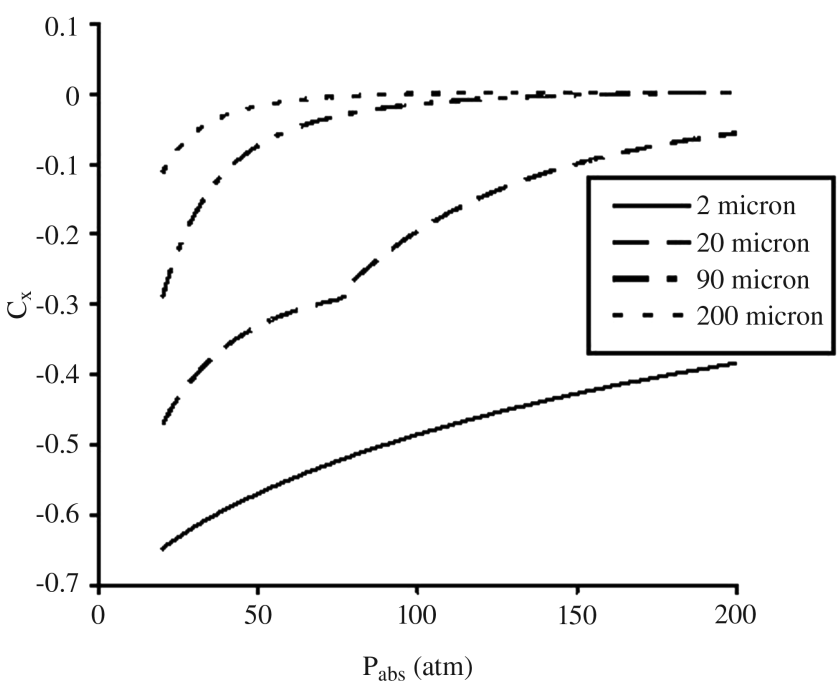

Fig. 4 Coupling coefficient $C_{x}$ for the Cohen and Strand model [5]. 


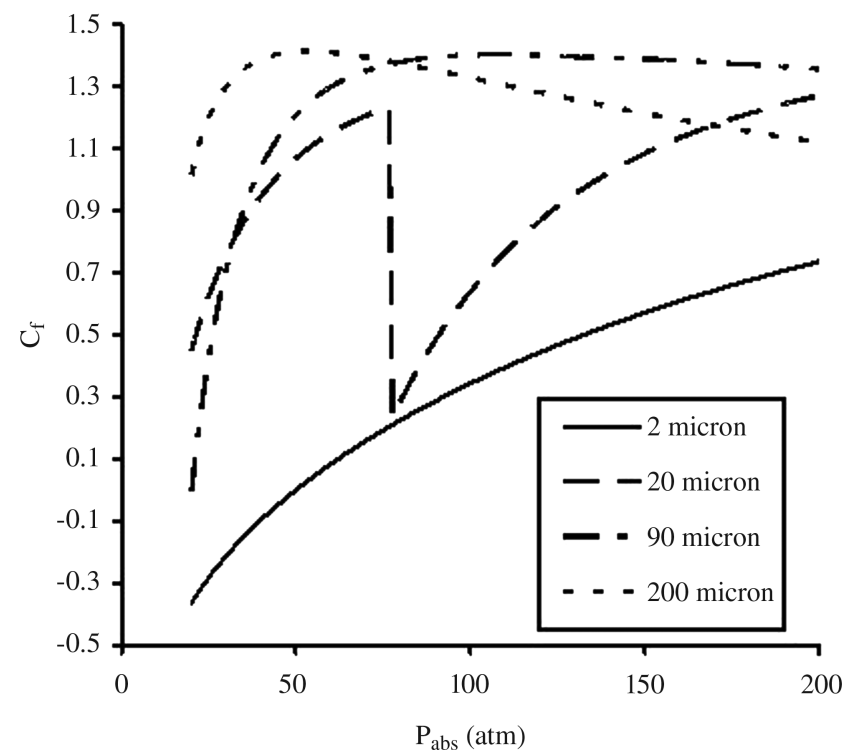

Fig. 5 Coupling coefficient $C_{f}$ for the Cohen and Strand model [ㅁ]

All of the parameters are naturally divided into four groups: 1) AP coefficients $A_{x}, B_{x}, n_{x} ; 2$ ) binder coefficients $A_{f}, B_{f}, n_{f} ; 3$ ) coupling coefficients $C_{x}, C_{f}$; and 4) frequency ratio $\delta$.

The influence of the AP coefficients turned out to be analogous to $A, B, n$ in the classical solution. The increase in $A_{x}$ increases the response and moves its maximum to higher frequencies. Decreasing $B_{x}$ makes the response function curve more peaked, whereas larger $n_{x}$ increases the value of the response but not its frequency dependence.

The influence of the binder coefficients is shown in Figs. 6-8. We see that for $A_{f}$ it is rather limited. One has to vary it considerably to achieve a small change. This is analogous to the small effect of binder decomposition kinetics on steady-state burning rate. Increasing $B_{f}$ makes the binder heat feedback smaller and therefore the AP response larger. A certain shift of the peak frequency to the right is also seen in Fig. 7. On the other hand, increasing $n_{f}$ decreases the AP response as more pressure-sensitive heating goes to the binder.

Figures 9 and 10 are plots of the response function for several $C_{x}$ and $C_{f}$. As expected, the AP response is larger for less negative $C_{x}$ and smaller $C_{f}$ (less coupling from or less coupling to the binder). No change in the forms of the curves can be observed in the figures. Again, we see the stabilizing influence of the binder.

Finally, Fig. 11 shows the influence of the frequency ratio $\delta$. What is interesting is that it is frequency dependent. Although for low frequencies $\left(\Omega_{1}<5\right)$, the response is larger for lower $\delta$, for high frequencies $\left(\Omega_{1}>20\right)$, it is larger for higher $\delta$. There is an intermediate region in between.

To understand the reason for this, one needs to observe that increasing $\delta$ for given $\Omega_{1}$ makes the binder frequency $\Omega_{2}$ higher. If $\Omega_{2}$ is small, it is shifted toward the peak area, and so the binder response becomes larger and the AP response correspondingly smaller. On the other hand, if $\Omega_{2}$ is higher than the peak frequency, then the binder response decreases and the AP response increases. Frequency ratio reflects differences in mean regression rates and thermal diffusivities between AP and binder. Again, we see the binder being more or less a stabilizing influence on the system.

\section{Intrinsic Instability}

It is well known that QSHOD-type models of homogeneous propellants have intrinsic stability limits, that is, there are regions of its parameters in which no steady burning is possible because the combustion is inherently unstable [2]. The goal of this section is to consider the possibility of intrinsic instability for the present model. We shall derive sufficient conditions to prevent intrinsic instability and use the Cohen and Strand model [5] to see if they are satisfied in practice.

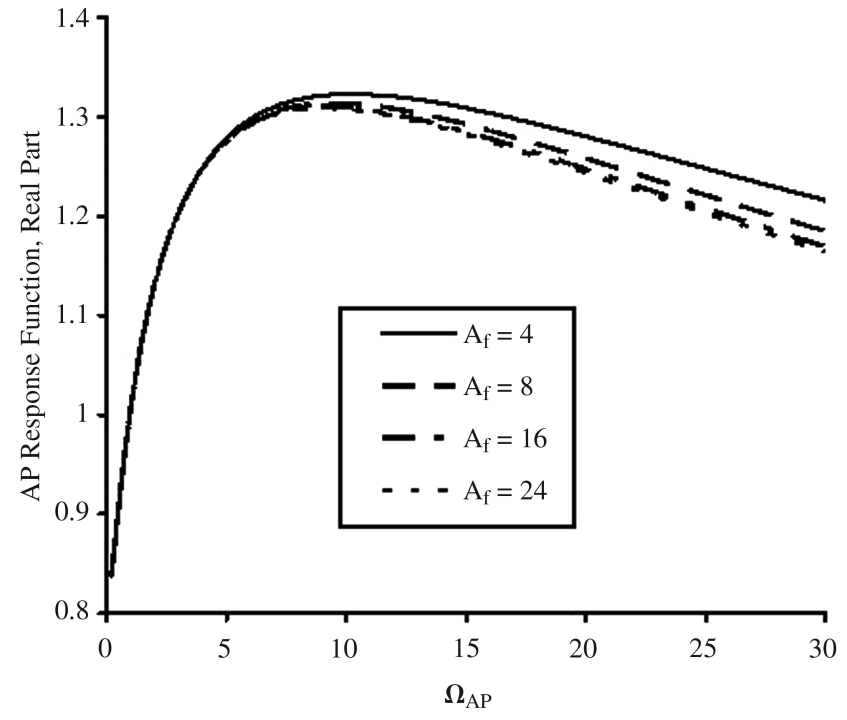

Fig. 6 Real part of AP response function for several $\boldsymbol{A}_{f}$.

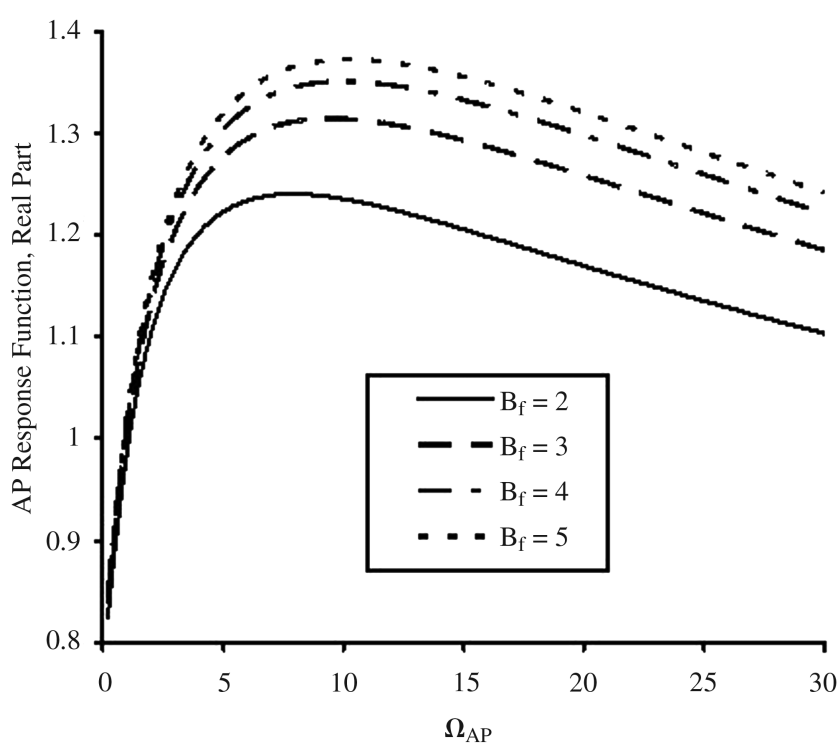

Fig. 7 Real part of AP response function for several $\boldsymbol{B}_{f}$.

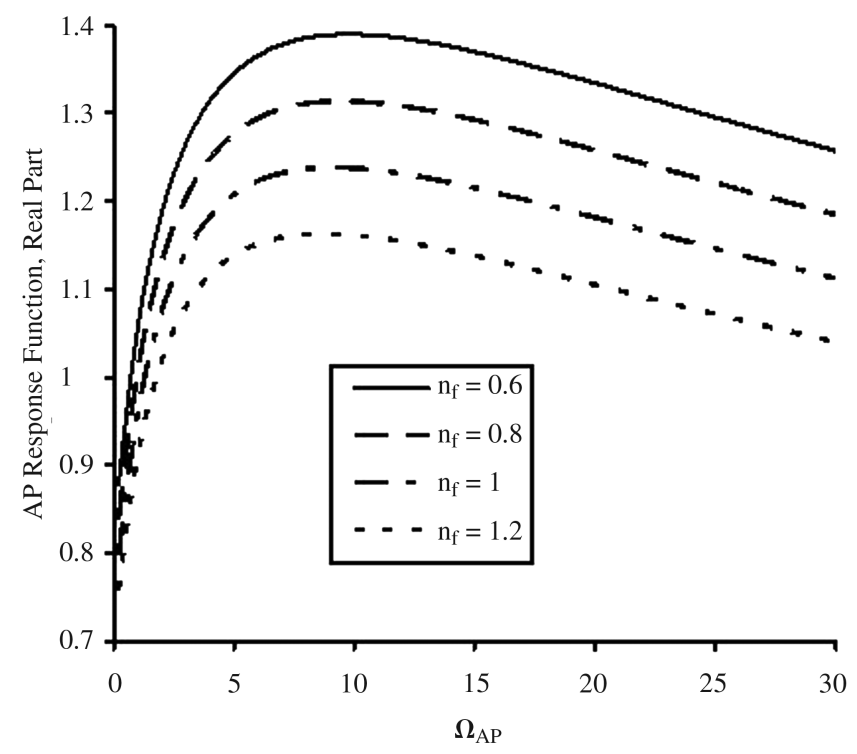

Fig. 8 Real part of AP response function for several $n_{f}$. 


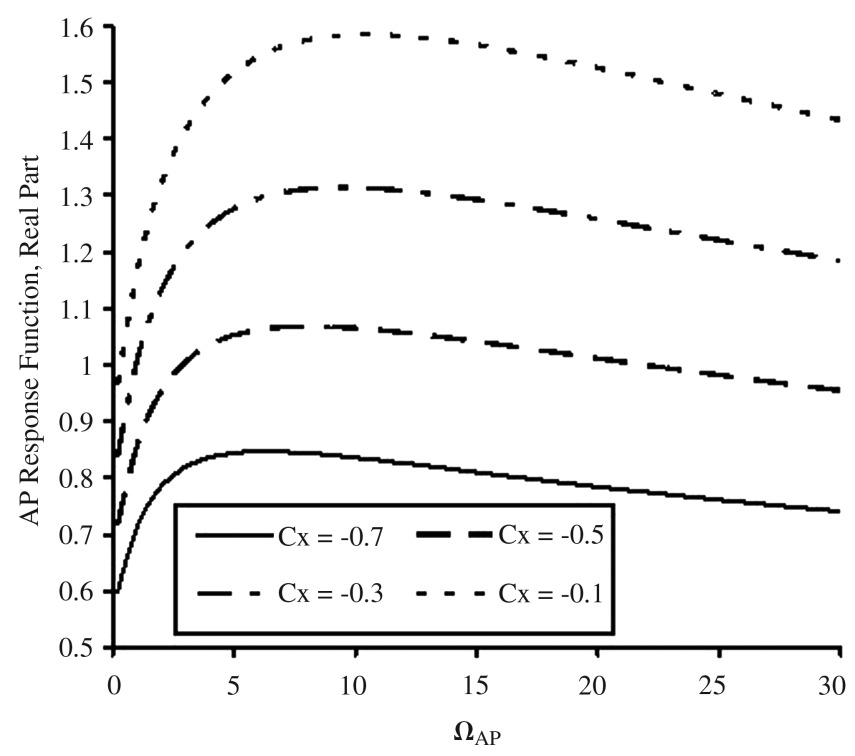

Fig. 9 Real part of AP response function for several $C_{x}$.

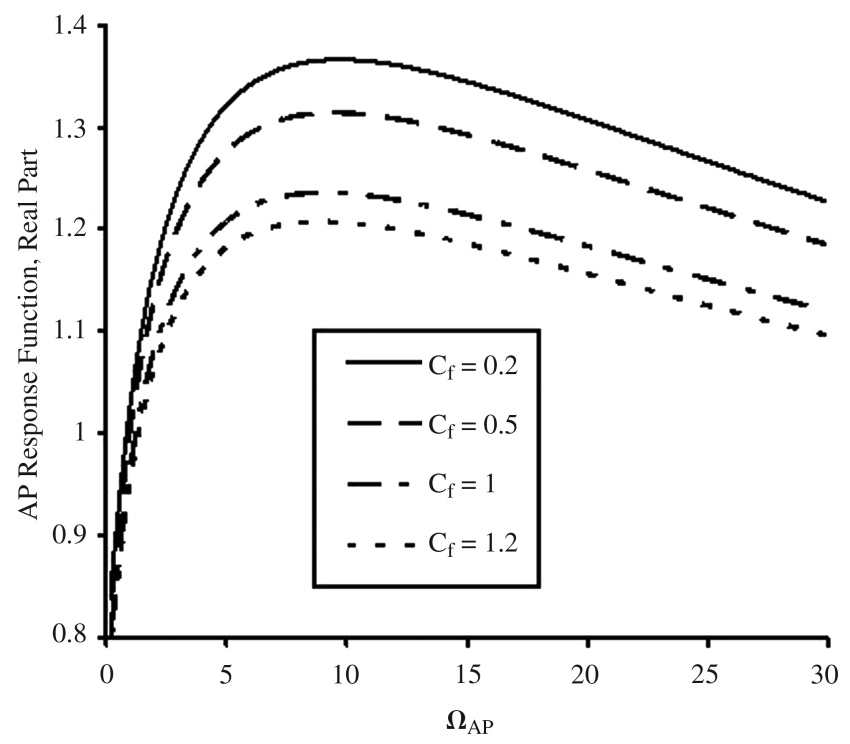

Fig. 10 Real part of AP response function for several $C_{f}$.

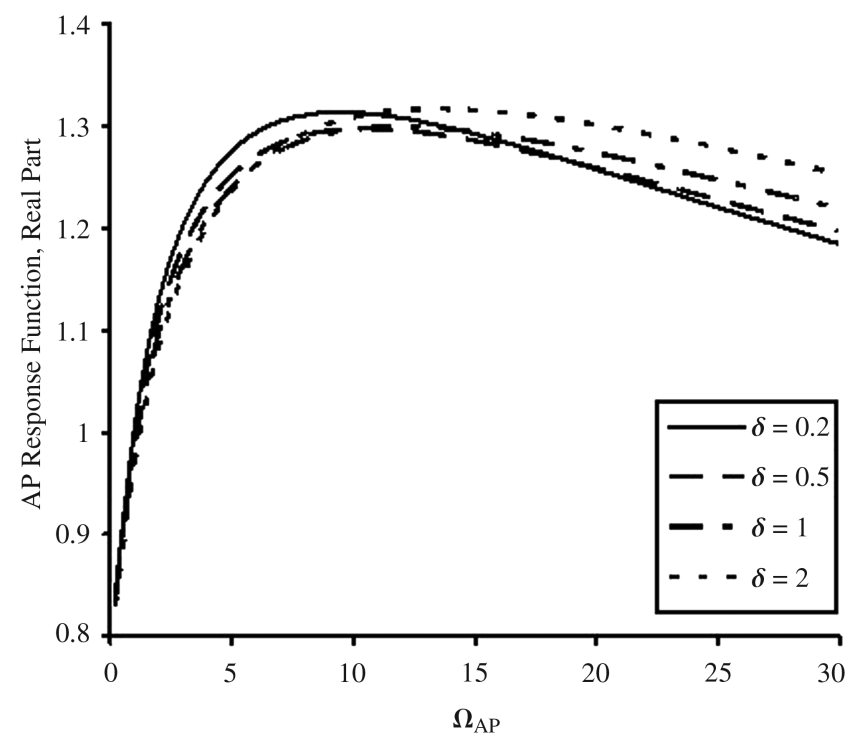

Fig. 11 Real part of AP response function for several $\delta$.
The solutions (15) and (16) can be written as

$$
\begin{aligned}
& R_{x}=\frac{n_{x} B_{x} D_{f}+C_{x} n_{f} B_{f}}{D_{x} D_{f}-C_{x} C_{f}} \\
& R_{f}=\frac{n_{f} B_{f} D_{x}+C_{f} n_{x} B_{x}}{D_{x} D_{f}-C_{x} C_{f}}
\end{aligned}
$$

Here,

$$
\begin{gathered}
D_{x}=\lambda / A_{x}+1 / \lambda-\left(1-B_{x}\right)-1 / A_{x} \\
D_{f}=\mu / A_{f}+1 / \mu-\left(1-B_{f}\right)-1 / A_{f}
\end{gathered}
$$

For intrinsic instability, the denominator in Eqs. (21) and (22) must vanish. This is equivalent to

$$
D_{x}^{(\mathrm{Re})} D_{f}^{(\mathrm{Re})}-D_{x}^{(\mathrm{Im})} D_{f}^{(\mathrm{Im})}-C_{x} C_{f}=0
$$

$$
D_{x}^{(\mathrm{Re})} D_{f}^{(\mathrm{Im})}+D_{x}^{(\mathrm{Im})} D_{f}^{(\mathrm{Re})}=0
$$

where $D_{x}^{(\mathrm{Re})}, D_{f}^{(\mathrm{Re})}, D_{x}^{(\mathrm{Im})}, D_{f}^{(\mathrm{Im})}$ are the real and imaginary parts of $D_{x}, D_{f}$.

We consider the case where both $D_{x}^{(\mathrm{Re})}, D_{f}^{(\mathrm{Re})}$ are positive:

$$
D_{x}^{(\mathrm{Re})}>0 ; \quad D_{f}^{(\mathrm{Re})}>0
$$

Then, to satisfy Eq. (26), we would need

$$
D_{x}^{(\mathrm{Im})} D_{f}^{(\mathrm{Im})}<0
$$

Comparing Eqs. (25) and (28) one sees that if Eq. (27) is satisfied then no intrinsic stability is possible for $C_{x} C_{f}<0$. If $\overline{C_{x}} C_{f}>0$ then, to prevent intrinsic instability, one needs to impose the additional condition

$$
D_{x}^{(\mathrm{Re})} D_{f}^{(\mathrm{Re})}>C_{x} C_{f}
$$

In Sec. III, we obtained that, for the Cohen and Strand model [5], $C_{x} C_{f}$ is almost always negative (see Figs. $\underline{4}$ and $\underline{5}$ ). This is consistent with the physics of the problem, as one would expect a destabilizing influence of the AP on the binder $\left(C_{f}>0\right)$ and a stabilizing influence of the inert binder on the $\mathrm{AP}\left(C_{x}<0\right)$. However, for fine AP and low pressure, $C_{x} C_{f}>0$ was obtained. Whether this is an artifact of the model or reflects the real physical situation is at present unclear. Nevertheless, we will consider both $C_{x} C_{f}<0$ and $C_{x} C_{f}>0$ in our analysis.

To obtain conditions for the parameters, we write the real parts explicitly:

$$
\begin{aligned}
D_{x}^{(\mathrm{Re})} & =\frac{\lambda_{r}-1}{A_{x}}+\frac{1}{2 \lambda_{\mathrm{Re}}-1}-\left(1-B_{x}\right) \\
D_{f}^{(\mathrm{Re})} & =\frac{\mu_{r}-1}{A_{f}}+\frac{1}{2 \mu_{\mathrm{Re}}-1}-\left(1-B_{f}\right)
\end{aligned}
$$

Here, $\lambda_{\operatorname{Re}}, \mu_{\mathrm{Re}}$ are the real parts of the complex frequencies $\lambda, \mu$ (see the Appendix). Equation (18) was used in obtaining Eqs. (30) and (31).

Calculating the minimum values of $D_{x}^{(\mathrm{Re})}, D_{f}^{(\mathrm{Re})}$ for $1 \leq \lambda_{\mathrm{Re}}<\infty$, $1 \leq \mu_{\operatorname{Re}}<\infty$, one sees that Eq. (27) is equivalent to

$$
B_{x}+\sqrt{\frac{2}{A_{x}}}-\frac{1}{2 A_{x}}>1
$$




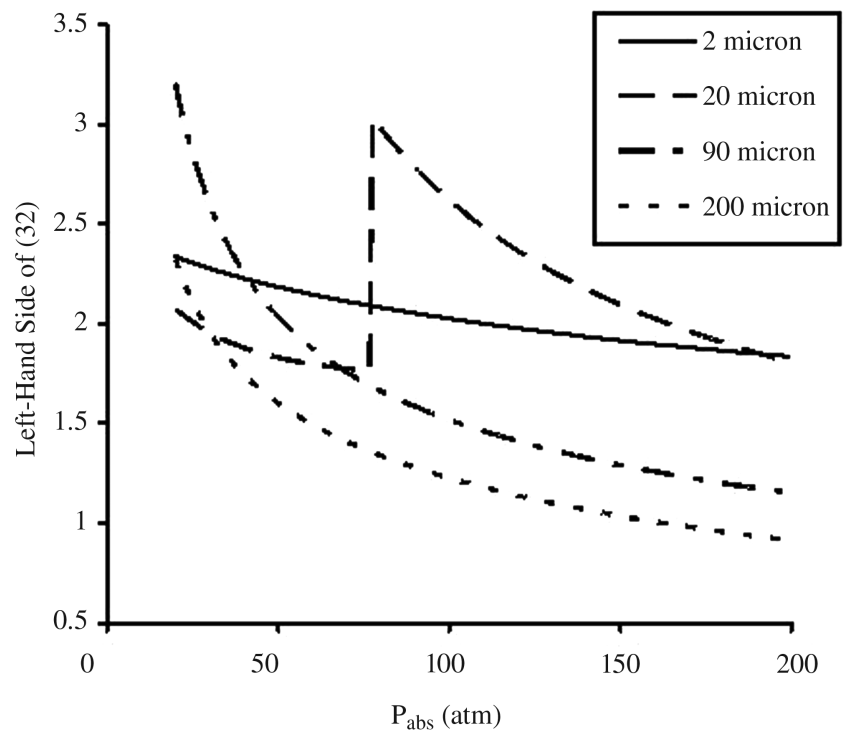

Fig. 12 Sufficient condition to prevent intrinsic instability.

$$
B_{f}+\sqrt{\frac{2}{A_{f}}}-\frac{1}{2 A_{f}}>1
$$

Equations (32) and (33) are the sufficient conditions to prevent intrinsic instability for $\bar{C}_{x} C_{f}<0$. If $C_{x} C_{f}>0$, another condition must be added:

$$
\left(B_{x}+\sqrt{\frac{2}{A_{x}}}-\frac{1}{2 A_{x}}-1\right)\left(B_{f}+\sqrt{\frac{2}{A_{f}}}-\frac{1}{2 A_{f}}-1\right)>C_{x} C_{f}
$$

To see if Eqs. (32-34) are satisfied in practice, we use the values of the coefficients for the Cohen and Strand model [5] calculated in Sec. IV (see Figs. 2-5). One sees from these figures that Eq. (33) is always satisfied and Eq. (34) is satisfied for $C_{x} C_{f}>0$. However, this is not the case for condition (32).

The left-hand side of condition (32) is plotted vs pressure for four particle sizes in Fig. 12. The figure shows that condition (32) is violated for high pressure and coarse AP. For $200 \mu \mathrm{m}$ AP, the limit value of pressure is slightly above $160 \mathrm{~atm}$. In numerical computations of composite propellant combustion [8], intrinsic instability was found at 170 atm. Hence, the criterion of condition (32) is not only a sufficient condition but probably close to the necessary condition as well.

It should be stressed that, for high pressure and coarse $\mathrm{AP}, C_{x} \approx 0$ and AP is practically decoupled from the binder and behaves like pure AP. Only under these conditions, the Cohen and Strand model [5] allows intrinsic instability. One can therefore reach a tentative conclusion that the stabilizing influence of an inert binder prevents an intrinsic instability under most conditions of interest. We show again the importance of avoiding coarse $\mathrm{AP}$ at high pressures, this time explained with mathematics.

\section{Pressure Exponents in Composite Propellants}

Pressure exponents represent the pressure dependence of the steady-state burning rate. For a homogeneous propellant, it is defined as

$$
n=\frac{\partial \ln \bar{r}}{\partial \ln \bar{p}}
$$

Similarly, for a composite propellant, one can define AP and binder pressure exponents that represent the pressure dependence of the steady-state burning rates $\bar{r}_{1}$ and $\bar{r}_{2}$ :

$$
n_{1}=\frac{\partial \ln \bar{r}_{1}}{\partial \ln \bar{p}} ; \quad n_{2}=\frac{\partial \ln \bar{r}_{2}}{\partial \ln \bar{p}}
$$

However, the situation for composite propellants turns out to be more complicated. In this section, we will demonstrate the existence of two types of pressure exponents for a composite propellant and discuss the physical reasons for this phenomenon.

\section{A. Two Types of Pressure Exponent}

The classical pressure exponent for a homogeneous propellant $n$ can be defined in two ways. Besides the usual definition [Eq. (35)], one can use the surface boundary condition (8) to define it as

$$
n=\left.\frac{\bar{p}}{B} \frac{\partial}{\partial p^{\prime}}\left[\left.\frac{\partial \theta^{\prime}}{\partial z}\right|_{z=0}\right]\right|_{r^{\prime}=0}
$$

The definitions of Eqs. (35) and (37) describe two aspects of the same phenomena. The former shows the pressure dependence of the steady-state burning rate, whereas the latter relates heat feedback and pressure oscillations. Burning rate is determined by surface temperature, which in turn is obtained from the heat flux boundary condition at the solid surface.

For a composite propellant, we have introduced two parameters $n_{x}$ and $n_{f}$ in boundary conditions (9) and (10). They can be defined by extending Eq. (37) to composite propellants

$$
\begin{aligned}
& n_{x}=\left.\frac{\bar{p}}{B_{x}} \frac{\partial}{\partial p^{\prime}}\left[\left.\frac{\partial \theta_{1}^{\prime}}{\partial z_{1}}\right|_{z_{1}=0}\right]\right|_{r_{1}^{\prime}=r_{2}^{\prime}=0} \\
& n_{f}=\left.\frac{\bar{p}}{B_{f}} \frac{\partial}{\partial p^{\prime}}\left[\left.\frac{\partial \theta_{2}^{\prime}}{\partial z_{2}}\right|_{z_{2}=0}\right]\right|_{r_{1}^{\prime}=r_{2}^{\prime}=0}
\end{aligned}
$$

However, there are other ways, besides Eqs. (38) and (39), to extend Eq. (37) to composite propellants. The definitions of Eqs. (38) and (39) assume that one calculates the $p^{\prime}$ derivative while keeping both $\bar{r}_{1}^{\prime}$ and $r_{2}^{\prime}$ at zero. However, it is not necessary to keep both of them at zero. We can extend Eq. (37) to a composite propellant while keeping each component's corresponding steady-state burning rate, that is,

$$
\begin{aligned}
& n_{1}=\lim _{f \rightarrow 0}\left\{\left.\frac{\bar{p}}{B_{x}} \frac{\partial}{\partial p^{\prime}}\left[\left.\frac{\partial \theta_{1}^{\prime}}{\partial z_{1}}\right|_{z_{1}=0}\right]\right|_{r_{1}^{\prime}=0}\right\} \\
& n_{2}=\lim _{f \rightarrow 0}\left\{\left.\frac{\bar{p}}{B_{f}} \frac{\partial}{\partial p^{\prime}}\left[\left.\frac{\partial \theta_{2}^{\prime}}{\partial z_{2}}\right|_{z_{2}=0}\right]\right|_{r_{2}^{\prime}=0}\right\}
\end{aligned}
$$

The limits in Eqs. (40) and (41) are needed in accordance with the steady-state limit.

To calculate $n_{1}$ and $n_{2}$, we use the surface boundary conditions (9) and (10) and the response function definition Eq. (14), to obtain from Eqs. (40) and (41)

$$
n_{1}=n_{x}+\left.\frac{C_{x}}{B_{x}} R_{f}\right|_{f=0} ; \quad n_{2}=n_{f}+\left.\frac{C_{f}}{B_{f}} R_{x}\right|_{f=0}
$$

As will be proven later [see Eq. (2)], it follows from Eq. (42) that

$$
n_{1}=n_{x}+\frac{C_{x}}{B_{x}} n_{2} ; \quad n_{2}=n_{f}+\frac{C_{f}}{B_{f}} n_{1}
$$

Substituting into Eq. (42) the zero-frequency limits of both response functions that can be retrieved from Eqs. (15) and (16), one obtains

$$
n_{1}=\frac{B_{f}\left(n_{x} B_{x}+C_{x} n_{f}\right)}{B_{x} B_{f}-C_{x} C_{f}}
$$




$$
n_{2}=\frac{B_{x}\left(n_{f} B_{f}+C_{f} n_{x}\right)}{B_{x} B_{f}-C_{x} C_{f}}
$$

\section{B. Method of Homogenization}

To understand the meaning of $n_{x}$ and $n_{f}$, we will use what one might call a "homogenization" of the propellant.ti Consider the AP surface boundary condition (9)

$$
\frac{\partial \theta_{1}^{\prime}}{\partial z_{1}}=n_{x} B_{x} \frac{p^{\prime}}{\bar{p}}+\left(1-B_{x}+\frac{1}{A_{x}}\right) \frac{r_{1}^{\prime}}{\bar{r}_{1}}+C_{x} \frac{r_{2}^{\prime}}{\bar{r}_{2}}
$$

By definition of the binder response function $R_{f}$,

$$
\frac{r_{2}^{\prime}}{\bar{r}_{2}}=R_{f} \frac{p^{\prime}}{\bar{p}}
$$

Hence, Eq. (46) can be written as

$$
\frac{\partial \theta_{1}^{\prime}}{\partial z_{1}}=n_{x}^{\prime} B_{x} \frac{p^{\prime}}{\bar{p}}+\left(1-B_{x}+\frac{1}{A_{x}}\right) \frac{r_{1}^{\prime}}{\bar{r}_{1}}
$$

where

$$
n_{x}^{\prime}=n_{x}\left(1+\frac{C_{x}}{n_{x} B_{x}} R_{f}\right)
$$

Equation (48) looks exactly like the surface boundary condition for a homogeneous propellant, Eq. ( ). Of course, it is an unusual homogeneous propellant because its pressure exponent $n_{x}^{\prime}$ depends on the oscillatory frequency through $R_{f}$. Nevertheless, the classical relationships remain valid for this propellant. For example, its response function $R_{x}$ is given by

$$
R_{x}=\frac{n_{x}^{\prime} A_{x} B_{x}}{\lambda+A_{x} / \lambda-1-A_{x}\left(1-B_{x}\right)}
$$

Because of $n_{x}^{\prime}$ being a function of $R_{f}$, Eq. (50) yields a relation between $\mathrm{AP}$ and binder response functions $R_{x}$ and $R_{f}$. One can use Eqs. (15) and (16) to verify Eq. (50). The same method can also be applied to the binder component, for which $n_{f}^{\prime}$ can be similarly defined.

Thus, we can make an important conclusion that each component in the composite propellant behaves like a homogeneous propellant with a frequency-dependent pressure exponent. The $n_{x}$ and $n_{f}$, therefore, are pressure exponents characteristic of the nonsteady coupling. For $C_{x}=0, n_{x}^{\prime}=n_{x}=n_{1}$. For $C_{f}=0, n_{f}^{\prime}=n_{f}=n_{2}$; the binder can still burn because of the steady-state heat feedback but there is no nonsteady interaction. The steady-state pressure exponents are obtained by taking the zero-frequency limit of $n_{x}^{\prime}$ and $n_{f}^{\prime}$, which yields Eq. (42) for $n_{1}$ and for $n_{2}$.

One sees from Eq. (43) that, without coupling,

$$
n_{1}=n_{x} \quad \text { if } C_{x}=0 ; \quad n_{2}=n_{f} \quad \text { if } C_{f}=0
$$

This is consistent with the result that, when one of the unsteady coupling coefficients vanishes, the corresponding response function reduces to its homogeneous form.

It can also be verified that, as for homogeneous propellant, the response function value for zero frequency is equal to the corresponding pressure exponent:

$$
R_{x}=n_{1} \quad \text { for } f=0 ; \quad R_{f}=n_{2} \quad \text { for } f=0
$$

\footnotetext{
${ }^{\dagger}$ The method of homogenization can be used to calculate the response function of a multicomponent propellant (see [15]).
}

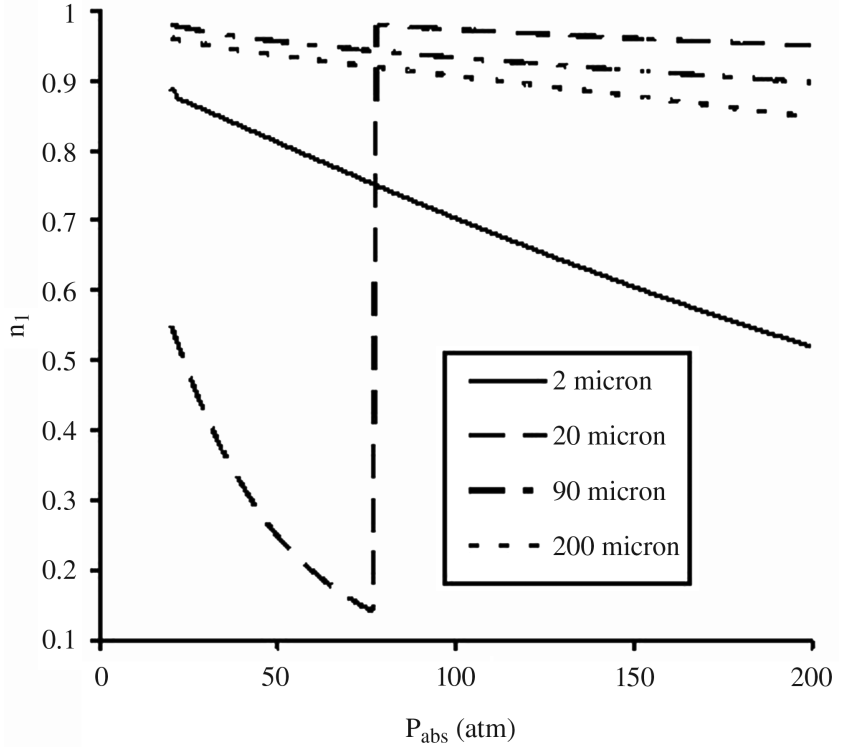

Fig. 13 AP pressure exponent in a propellant.

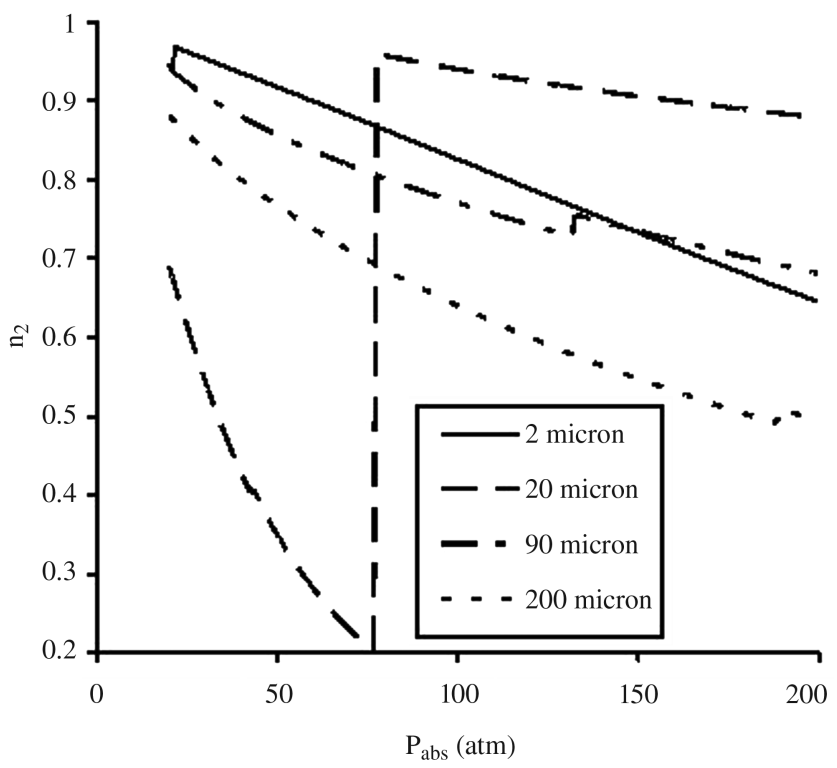

Fig. 14 Binder pressure exponent in a propellant.

\section{Practical Values}

To estimate the physically reasonable values of the pressure exponents, we calculated them for the Cohen and Strand model [5]. As in Sec. III, AP concentration was kept at $87 \%$ throughout the calculations and propellant bulk temperature was $25^{\circ} \mathrm{C}$.

Both pressure exponents $n_{1}$ and $n_{2}$ are plotted in Figs. 13 and 14 . One can see that the dependencies of $n_{1}$ and $n_{2}$ are more or $\overline{\text { less like }} \bar{n}_{x}$ and $n_{f}$. However, $n_{1}<n_{x}$ because $C_{x}<0$ and $n_{2}>n_{f}$ because $C_{f}>0$ [see Figs. $\underline{4}$ and $\underline{5}$ and Eq. (43)].

One can also define the pressure exponent for the whole propellant:

$$
n_{\text {prop }}=\frac{\partial \ln \bar{r}_{\text {prop }}}{\partial \ln \bar{p}}
$$

The total propellant burning rate is given by

$$
\bar{r}_{\text {prop }}=\frac{\rho_{x} S_{x} \bar{r}_{1}+\rho_{f} S_{f} \bar{r}_{2}}{\rho_{\text {prop }}}
$$


Here, $\rho_{x}, \rho_{f}$, and $\rho_{\text {prop }}$ are AP, binder, and propellant densities; $S_{x}$ and $S_{f}$ are fractions of propellant surface area occupied by AP and binder, respectively. This also yields the propellant response function from the component contributions.

Differentiating Eq. (54) with respect to $\ln \bar{p}$ and using Eq. (36), we obtain

$$
n_{\text {prop }}=\alpha n_{1}+(1-\alpha) n_{2}
$$

where $\alpha$ is the fraction of propellant mass flow rate from AP. The calculation shows [15] that $n_{\text {prop }}$ is high for fine and coarse particles but can be considerably smaller for intermediate sizes where diffusion control is important in the flame.

\section{Conclusions}

We have obtained a general analytical solution for the pressurecoupled response of a monomodal composite propellant, applied to AP and inert binder. The solution depends on the AP coefficients $A_{x}$, $B_{x}$, and $n_{x}$, the binder coefficients $A_{f}, B_{f}$, and $n_{f}$, the coupling coefficients $C_{x}$ and $C_{f}$, and the frequency ratio $\delta$. The influence of these parameters on the real part of the AP response function in the propellant has been investigated.

The influence of the AP coefficients is analogous to the $A, B$, and $n$ parameters of the classical homogeneous propellant solution. The increase in $A_{x}$ increases the response and moves its maximum to higher frequencies. Decreasing $B_{x}$ makes the response function curve more peaked, whereas larger $n_{x}$ increases the value of the response but not its frequency dependence.

The influence of $A_{f}$ was found to be limited due to the small effect of binder decomposition kinetics on burning rate. Increasing $B_{f}$ makes the binder heat feedback smaller and therefore the AP response larger, whereas increasing $n_{f}$ decreases the AP response as more heat goes to the binder.

Less coupling from the binder (less negative $C_{x}$ ) or less coupling to the binder (smaller $C_{f}$ ) increases the response. The influence of the frequency ratio $\delta$ was found to be frequency dependent.

The parameters $A_{x}, A_{f}, B_{x}, B_{f}, n_{x}, n_{f}, C_{x}$, and $C_{f}$ were calculated for a particular case of the Cohen and Strand model [5]. The analytical solution was shown to be in excellent agreement with numerical computation results, and has the advantage of revealing important mechanisms. Thus, we see the destabilizing influence of the monopropellant AP and the stabilizing influence of the inert binder in the system.

The influence of absolute pressure and AP particle size on the parameters has been studied. $B_{x}$ in a propellant turned out to be much higher than in the classical formulation due to the stabilizing influence of the binder in the coupling. $B_{x}$ decreases with increasing pressure mainly because of decreasing flame heights.

The $B_{f}$ parameter was found to be even larger than $B_{x}$. This shows that an inert binder is highly stabilizing to the propellant system. Because of the binder being heated only by the diffusion flame, $n_{f}$ decreases much faster than $n_{x}$ with increasing pressure.

The AP coupling coefficient $C_{x}$ is always negative. This reflects the stabilizing influence of the binder on the AP by the coupling. However, $C_{x}$ becomes less negative with increasing pressure and particle size. $C_{x}$ vanishes for high pressures and large sizes, where the AP becomes decoupled from the binder and behaves more like pure AP. The binder coupling coefficient $C_{f}$ is generally positive, showing that the AP has a destabilizing influence on the binder. The AP monopropellant combustion is the destabilizing influence on the system, whereas the diffusion flame with binder is stabilizing.

Sufficient conditions preventing intrinsic instability in a composite propellant were obtained. Using the Cohen and Strand model [5], we demonstrated that these conditions are satisfied in most practical situations. In agreement with previous numerical results, it was found mathematically that intrinsic instability is possible only at high pressures and with coarse AP. At this condition, AP is practically decoupled from the binder and behaves like pure AP. Thus, it appears that in most practical cases, the stabilizing influence of an inert binder prevents intrinsic instability. To show this, it is necessary to treat the two-component system as it really is rather than as an averaged one-component system.

We have shown that, for a two-component composite propellant, two forms of pressure exponents arise from the analysis. Whereas the conventional $n_{1}$ and $n_{2}$ show the pressure dependence of both AP and binder steady-state burning rates, $n_{x}$ and $n_{f}$ arise from the oscillations of the unsteady heat feedback and unsteady coupling. The general expressions for $n_{1}$ and $n_{2}$ in propellants have been obtained and their dependencies on pressure and AP particle size were studied for the Cohen and Strand model [5]. In addition, the pressure exponent of the aggregate composite propellant was defined. We also demonstrated that both AP and binder in the composite propellant system behave like a homogeneous propellant with a frequency-dependent pressure exponent characteristic of the nonsteady coupling.

The convenience of an analytical solution will facilitate further studies of the response function of composite propellants. Other advanced energetic ingredients may be more destabilizing than AP and can be evaluated for linearized analysis by studying the monopropellants themselves in the framework of this theoretical approach, which began with the study of AP [14].

\section{Appendix: Real and Imaginary Parts of Binder and Ammonium Perchlorate Response Functions}

The real and imaginary parts of the binder and AP response functions $R_{f}$ and $R_{x}$ are as follows:

$$
\begin{aligned}
& R_{x}=\frac{e_{1} e_{5}+e_{2} e_{6}}{e_{5}^{2}+e_{6}^{2}}+i \frac{e_{2} e_{5}-e_{1} e_{6}}{e_{5}^{2}+e_{6}^{2}} \\
& R_{f}=\frac{e_{3} e_{5}+e_{4} e_{6}}{e_{5}^{2}+e_{6}^{2}}+i \frac{e_{4} e_{5}-e_{3} e_{6}}{e_{5}^{2}+e_{6}^{2}}
\end{aligned}
$$

Here,

$$
e_{1}=n_{x} B_{x}\left[\mu_{\mathrm{Re}}\left(\frac{1}{A_{f}}+\frac{1}{\mu_{\mathrm{Re}}^{2}+\mu_{\mathrm{Im}}^{2}}\right)-\left(1-B_{f}+\frac{1}{A_{f}}\right)\right]+n_{f} B_{f} C_{x}
$$

$$
e_{2}=n_{x} B_{x} \mu_{\mathrm{Im}}\left(\frac{1}{A_{f}}-\frac{1}{\mu_{\mathrm{Re}}^{2}+\mu_{\mathrm{Im}}^{2}}\right)
$$

$$
e_{3}=n_{f} B_{f}\left[\lambda_{r}\left(\frac{1}{A_{x}}+\frac{1}{\lambda_{\mathrm{Re}}^{2}+\lambda_{\mathrm{Im}}^{2}}\right)-\left(1-B_{x}+\frac{1}{A_{x}}\right)\right]+n_{x} B_{x} C_{f}
$$

$$
e_{4}=n_{f} B_{f} \lambda_{\mathrm{Im}}\left(\frac{1}{A_{x}}-\frac{1}{\lambda_{\mathrm{Re}}^{2}+\lambda_{\mathrm{Im}}^{2}}\right)
$$

$$
\begin{aligned}
e_{5} & =\left[\lambda_{\mathrm{Re}}\left(\frac{1}{A_{x}}+\frac{1}{\lambda_{\mathrm{Re}}^{2}+\lambda_{\mathrm{Im}}^{2}}\right)-\left(1-B_{x}+\frac{1}{A_{x}}\right)\right] \\
& \times\left[\mu_{\mathrm{Re}}\left(\frac{1}{A_{f}}+\frac{1}{\mu_{\mathrm{Re}}^{2}+\mu_{\mathrm{Im}}^{2}}\right)-\left(1-B_{f}+\frac{1}{A_{f}}\right)\right] \\
& -\lambda_{\mathrm{Im}} \mu_{\mathrm{Im}}\left(\frac{1}{A_{x}}-\frac{1}{\lambda_{\mathrm{Re}}^{2}+\lambda_{\mathrm{Im}}^{2}}\right)\left(\frac{1}{A_{f}}-\frac{1}{\mu_{\mathrm{Re}}^{2}+\mu_{\mathrm{Im}}^{2}}\right)-C_{x} C_{f}
\end{aligned}
$$




$$
\begin{aligned}
e_{6} & =\lambda_{\mathrm{Im}}\left(\frac{1}{A_{x}}-\frac{1}{\lambda_{\mathrm{Re}}^{2}+\lambda_{\mathrm{Im}}^{2}}\right) \\
& \times\left[\mu_{\mathrm{Re}}\left(\frac{1}{A_{f}}+\frac{1}{\mu_{\mathrm{Re}}^{2}+\mu_{\mathrm{Im}}^{2}}\right)-\left(1-B_{f}+\frac{1}{A_{f}}\right)\right] \\
& +\mu_{\mathrm{Im}}\left(\frac{1}{A_{f}}-\frac{1}{\mu_{\mathrm{Re}}^{2}+\mu_{\mathrm{Im}}^{2}}\right) \\
& \times\left[\lambda_{\mathrm{Re}}\left(\frac{1}{A_{x}}+\frac{1}{\lambda_{\mathrm{Re}}^{2}+\lambda_{\mathrm{Im}}^{2}}\right)-\left(1-B_{x}+\frac{1}{A_{x}}\right)\right]
\end{aligned}
$$

The real and imaginary parts of the complex frequencies $\lambda$ and $\mu$ are given by

$$
\begin{gathered}
\lambda_{\mathrm{Re}}=\frac{1}{2}\left[1+\left(\frac{\sqrt{1+16 \Omega_{1}^{2}}+1}{2}\right)^{1 / 2}\right] \\
\lambda_{\mathrm{Im}}=\frac{1}{2}\left(\frac{\sqrt{1+16 \Omega_{1}^{2}}-1}{2}\right)^{1 / 2} \\
\mu_{\mathrm{Re}}=\frac{1}{2}\left[1+\left(\frac{\sqrt{1+16 \Omega_{2}^{2}}+1}{2}\right)^{1 / 2}\right] \\
\mu_{\mathrm{Im}}=\frac{1}{2}\left(\frac{\sqrt{1+16 \Omega_{2}^{2}}-1}{2}\right)^{1 / 2}
\end{gathered}
$$

\section{Acknowledgments}

This work was sponsored partly by the California Institute of Technology and partly by the U.S. Office of Naval Research Multidisciplinary University Research Initiative under Grant No. N00014-95-I-1338. Judah Goldwasser was the Navy Program Manager. The supplemental support under Multidisciplinary University Research Initiative Grant No. N00014-95-I-1339, through the courtesy of Herman Krier, University of Illinois at Urbana-Champaign, is gratefully acknowledged.

\section{References}

[1] Price, E. W., and Flandro, G. A., "Status and Prospects for Future Developments," Nonsteady Burning and Combustion Stability of Solid Propellants, edited by L. De Luca, E. W. Price, and M. Summerfield, Vol. 143, Progress in Astronautics and Aeronautics, AIAA, Washington, D.C., 1992, pp. 849-873.
[2] Culick, F. E. C., "Review of Calculations for Unsteady Burning of a Solid Propellant," AIAA Journal, Vol. 6, No. 12, 1968, pp. 2241-2255. doi: $10.2514 / 3.4980$

[3] Nachbar, W., "Theoretical Study of the Burning of a Solid Propellant Sandwich," Solid Propellant Rocket Research, edited by M. Summerfield, Vol. 1, ARS Progress in Astronautics and Rocketry, Academic Press, New York, 1960, pp. 207-226.

[4] Price, E. W., Sambamurthi, J. K., Sigman, R. K., and Panyam, R. R., "Combustion of Ammonium Perchlorate-Polymer Sandwiches," Combustion and Flame, Vol. 63, No. 3, 1986, pp. 381-413. doi:10.1016/0010-2180(86)90007-6

[5] Cohen, N. S., and Strand, L. D., "Improved Model for the Combustion of AP Composite Propellants," AIAA Journal, Vol. 20, No. 12, 1982, pp. $1739-1746$ doi:10.2514/3.8013

[6] Cohen, N. S., and Strand, L. D., "Combustion Response to Compositional Fluctuations," AIAA Journal, Vol. 23, No. 5, 1985, pp. 760-767. doi: $10.2514 / 3.8981$

[7] Cohen, N. S., and Strand, L. D., "Effect of AP Particle Size on Combustion Response to Crossflow," AIAA Journal, Vol. 23, No. 5, 1985, pp. 776-780. doi: $10.2514 / 3.8983$

[8] Shusser, M., Culick, F. E. C., and Cohen, N. S., "Combustion Response of Ammonium Perchlorate Composite Propellants," Journal of Propulsion and Power, Vol. 18, No. 5, 2002, pp. 1093-1100.

[9] Rasmussen, B., and Frederick, R. A., Jr., "Nonlinear Hterogeneous Model of Composite Solid Propellant Combustion," Journal of Propulsion and Power, Vol. 18, No. 5, 2002, pp. 1086-1092.

[10] Surzhikov, S. T., Murphy, J. J., and Krier, H., "Two-Dimensional Model for Unsteady Burning Heterogeneous AP/Binder Solid Propellants," AIAA Paper 2000-3573, July 2000.

[11] Jackson, T. L., and Buckmaster, J., "Heterogeneous Propellant Combustion," AIAA Journal, Vol. 40, No. 6, 2002, pp. 1122-1130.

[12] Massa, L., Jackson, T. L., Buckmaster, J., and Campbell, M., "ThreeDimensional Heterogeneous Propellant Combustion," Proceedings of the Combustion Institute, Vol. 29, No. 2, 2002, pp. 2975-2983. doi:10.1016/S1540-7489(02)80363-7

[13] Brown, R. S., and Muzzy, R. J., "Linear and Nonlinear Pressure Coupled Combustion Instability of Solid Propellants," AIAA Journal, Vol. 8, No. 8, 1970, pp. 1492-1500. doi: $10.2514 / 3.5929$

[14] Shusser, M., Culick, F. E. C., and Cohen, N. S., "Combustion Response of Ammonium Perchlorate," AIAA Journal, Vol. 40, No. 4, 2002, pp. $722-730$.

[15] Shusser, M., and Culick, F. E. C., "Analytical Solution for Composite Solid Propellant Response Function," Guggenheim Jet Propulsion Center, California Inst. of Technology, Rept. CI 01-02, May 2001.

[16] Beckstead, M. W., Derr, R. L., and Price, C. F., "Model of Composite Solid-Propellant Combustion Based on Multiple Flames," AIAA Journal, Vol. 8, No. 12, 1970, pp. 2200-2207. doi: $10.2514 / 3.6087$ 